Patentregister

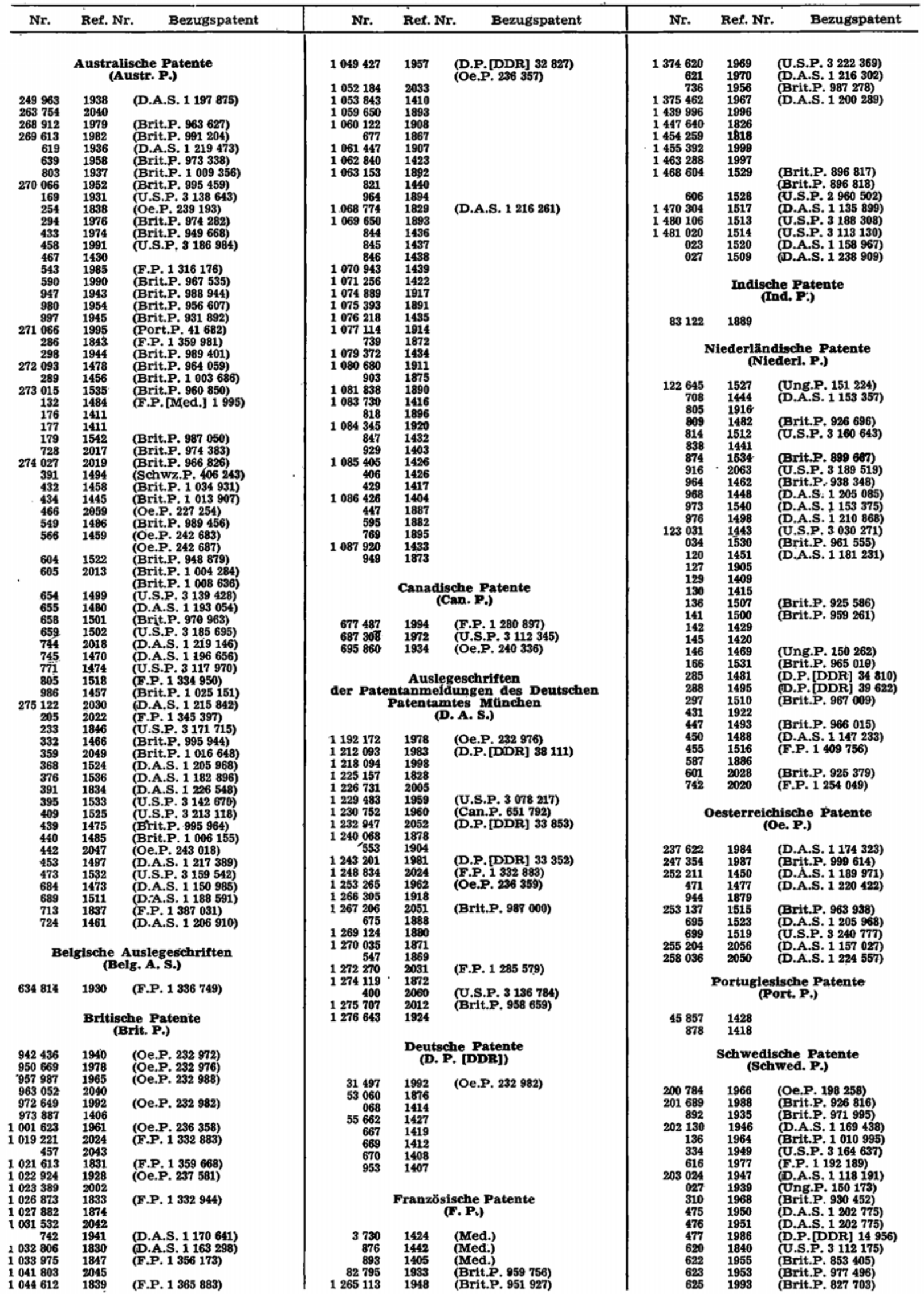




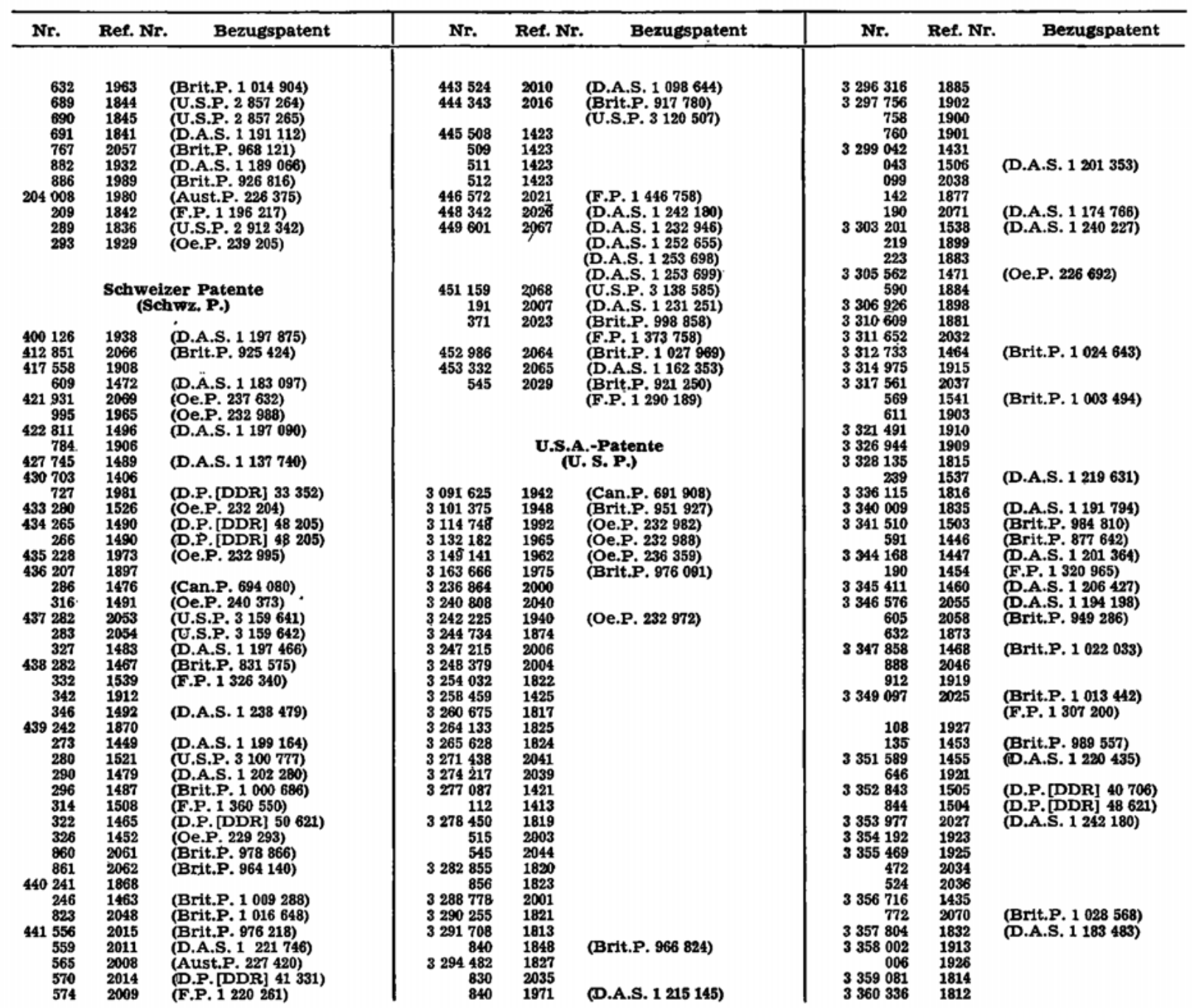




\section{Pharmazeutische Chemie}

(Wissenschaftliche Taschenbücher, Reihe Chemie)

3., verbesserte Auflage

1968. Etwa 277 Seiten $\cdot 8^{\circ} \cdot 8,-M$

Die Verfasser behandeln in diesem Band die Chemie der wichtigen Arzneimittel. Trotz der durch die gebotene Kürze bedingten Beschränkung auf die für eine moderne Therapie wesentlichsten Arzneimittel wurde eine einfache Aufzăhlung vermieden. Um die Entwicklung des Arzneischatzes zusammenhängend darstellen zu können, wurde der Stoff nach pharmakologischen Gesichtspunkten gegliedert. Gelegentliche pharmakologische Hinweise sollen dem medizinisch wèniger geschulten Leser ein besseres Verstăndnis ermöglichen. Bei den wichtigsten Arzneimitteln - meist synthetische organische Verbindungen der Naturstoffe wird nicht nur auf die chemische Struktur und die Wirkung, sondern auch auf Möglichkeiten der synthetischen Darstellung bzw. Isolierung, der Analytik und der Stabilitằt eingegangen. Zur Bezeichnung der Arzneimittel, die meist zahlreiche Namen besitzen, wurden weitgehend die von der Weltgesundheitsorganisation empfohlenen, gesetzlich nicht schutzfahigen internationalen Kurzbezeichnungen herangezogen. Daneben sind in der Regel noch einige Synonyma angegeben, wobei eimmal die Namen entsprechender Präparate der DDR und zum anderen die Namen, unter denen die Substanz bekannt geworden ist, ausgewăhlt wurden.

Bestellungen durch eine Buchhandlung erbeten

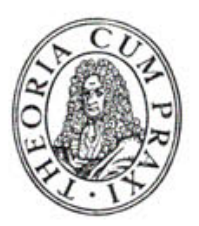

$\begin{array}{lllllllllllllllllllll}\mathbf{A} & \mathbf{K} & \mathbf{A} & \mathbf{D} & \mathbf{E} & \mathbf{M} & \mathbf{I} & \mathbf{E} & -\mathbf{V} & \mathbf{E} & \mathbf{R} & \mathbf{L} & \mathbf{A} & \mathbf{G} & \boldsymbol{B} & \mathbf{E} & \mathbf{R} & \mathbf{L} & \mathbf{I} & \mathbf{N}\end{array}$ 
Prof. Dr. WERNER HOLZMULLER / Dr. KURT ALTENBURG

\section{Physik der Kunststoffe}

Eine Einführung in das Verhalten makromolekularer Substanzen

1961 - XVI, 652 Seiten - 373 Abbildungen, davon 13 Abbildungen auf 7 Tafeln 38 Tabellen, davon 2 auf 2 Falttafeln $\cdot g r .8^{\circ} \cdot$ Lederin $62,-M$

Bisher gab es nur wenig zusammenfassende und keine einführenden Darstellungen in das Gebiet der synthetisch hergestellten organischen Werkstoffe. Aus diesem Grunde wurde dieses Lehrbuch abgefaBt, in dem einleitend die Prinzipien des Aufbanes makromolekularer Stoffe behandelt werden, soweit das für ein Verständnis des physikalischen Verhaltens der Hochpolymeren erforderlich ist. Anschließend wird das Verhalten makromolekularer Lösungen, vor allem im Hinblick auf die Methoden der Molëkulargewichtsbestimmung, diskutiert. Der gröBte Teil des Buches ist den Eigenschaften und dem Verhalten der Hochpolymeren in festem Zustand gewidmet. Nach Betrachtungen über die Struktur, die Kräfte und Ordnungszustände in festen Hochpolymeren und die Methoden ihrer Erforschung werden das mechanische Verhalten von seiten der phänomenologischen Theorie erörtert und eine Deutung der elastischen und plastischen Eigenschaften auf Grund der Platzwechseltheorie gegeben. Ferner werden die thermischen Eigenschaften der Kunststoffe untersucht. Im Hinblick auf die groBe praktische Bedentung der Kunststoffe als Isolierstoffe werden die elektrischen Eigenschaften ausführlich behandelt. Neben einer phänomenologischen Beschreibung des elektrischen Verhaltens werden die dielektrischen Eigenschaften vom molekularen Standpunkt aus diskutiert. Weiter werden die optischen Eigenschaften der Hochpolymeren, insbesondere die Anwendungsmöglichkeit der Ultrarotspektroskopie zur Aufklärung der Struktur und der vorhandenen Bindungen untersucht. Ein abschließender Uberblick zeigt die wichtigsten Prüfverfahren für Kunststoffe.

Bestellungen durch eine Buchhandlung erbeten

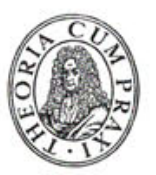
$\begin{array}{lllllllllllllllllllllll}\mathbf{A} & \mathbf{K} & \mathbf{A} & \mathbf{D} & \mathbf{E} & \mathbf{M} & \mathbf{I} & \mathbf{E} & - & \mathbf{V} & \mathbf{E} & \mathbf{R} & \mathbf{L} & \mathbf{A} & \mathbf{G} & & \mathbf{B} & \mathbf{E} & \mathbf{R} & \mathbf{L} & \mathbf{I} & \mathbf{N}\end{array}$ 